Research Paper

\title{
The relationship between miR-302b and EphA2 and their clinical significance in gastric cancer
}

\author{
Lanhua Tang ${ }^{*}$, Huabin $\mathrm{Hu}^{2 *}$, Yijing He ${ }^{3}$, Howard L. Mcleod ${ }^{3,4,5}$, Desheng Xiao ${ }^{6}$, Pan Chen ${ }^{7}$, Liangfang
}

Shen ${ }^{1}$,Shan Zeng ${ }^{1}$, Xianli Yin ${ }^{8}$, Jie Ge ${ }^{9}$, Li Li ${ }^{1}$, Jian Ma ${ }^{10}$, Zihua Chen ${ }^{9}$, Jin Huang ${ }^{1}$

1. Department of Oncology, Xiangya Hospital, Central South University, Changsha, 410008, China

2. The Sixth Affiliated Hospital of Sun Yat-Sen University, Guangzhou, 510655, China

3. Department of Dermatology, XiangYa Hospital, Central South University, Changsha, 410008, China

4. Department of Clinical Pharmacology, XiangYa Hospital, Central South University, Changsha, 410008, China

5. Moffitt Cancer Center, DeBartolo Family Personalized Medicine Institute, Tampa, FL 33612, USA

6. Department of Pathology, Xiangya Hospital, Central South University, Changsha, 410008, China

7. Department of Hepatobiliary Surgery, Hunan Cancer Hospital and The Affiliated Cancer Hospital of Xiangya School of Medicine, Central South

University, Changsha, 410013, China

8. Department of gastroenterology and urology, Hunan Cancer Hospital and The Affiliated Cancer Hospital of Xiangya School of Medicine, Central South University, Changsha, 410013,China

9. Department of General Surgery, Xiangya Hospital, Central South University, Changsha, 410008, China

10. Cancer Research Institute, Hunan Key Laboratory of Nonresolving Inflammation and Cancer, Central South University, Changsha, 410008, China.

* Lanhua Tang and Huabin Hu contributed equally to this work

$\square$ Corresponding author: Jin Huang, Department of Oncology, Xiangya Hospital, Central South University, Changsha, 410008, China; E-mail: jinhuang@csu.edu.cn

( ) Ivyspring International Publisher. This is an open access article distributed under the terms of the Creative Commons Attribution (CC BY-NC) license (https://creativecommons.org/licenses/by-nc/4.0/). See http://ivyspring.com/terms for full terms and conditions.

Received: 2018.01.30; Accepted: 2018.06.16; Published: 2018.08.06

\begin{abstract}
Introduction: EphA2 is a crucial oncogene in gastric cancer (GC) development and metastasis, and miR-302b can target EphA2 in gastric cancer. This study plans to investigate their relationship and clinical significance in clinical samples.

Materials and Methods: We explored the correlation of the expression of EphA2 and miR-302b, and their clinical significance in the training $(n=226)$ cohort of $G C$ patients, and then validated the results in the validation $(n=128)$ cohort.

Results: miR-302b was remarkably downregulated in GC tissues, while high EphA2 expression were detected, and they were inversely correlated both in mRNA and protein, $(r=-0.4209$, $P<0.0001 ; r=-0.336, P<0.001$, respectively). Furthermore, the pattern of high EphA2 and low miR-302b expression were found to be associated with poor overall survival in stage IV GC patients in both training and validation cohort.

Conclusions: The expression of miR-302b and EphA2 was inversely correlated, and had prognostic significance on GC in clinic.
\end{abstract}

Key words: miR-302b, EphA2, gastric cancer

\section{Introduction}

Gastric cancer is a malignant cancer with high incidence and mortality rate worldwide [1]. Although great achievements were made in the screening, most patients were diagnosed with inoperable advanced or metastatic gastric cancer. And the 5-year survival of advanced or metastatic gastric cancer is around 5-20\% [2]. Thus, it's worthwhile to explore the mechanism of gastric cancer progression and metastasis.
As a member of Receptor tyrosine kinases (RTKs) family, EphA2 functions in bi-directional signal transduction $[3,4]$. In normal cells, EphA2 is usually expressed at a low level; however, it's overexpressed in malignant tumor cells including gastric cancer [5-7]. In our previous study, we have demonstrated that EphA2 promotes epithelialmesenchymal transition (EMT) via Wnt/ $\beta$-catenin 
pathway in gastric cancer in vivo and in vitro and has important prognostic value for gastric cancer patients $[5,8,9]$, which supported that EphA2 is a potential target for the treatment of gastric cancer. However, the detailed mechanism of the regulation of EphA2 is still unknown.

MicroRNAs (miRNAs), are a class of non-coding RNAs with a small size of approximately 21-nt, and they mostly bind to the $3^{\prime}$-untranslated region (3'-UTR) of their target mRNAs, it is estimated that $30 \%$ of all protein-coding genes are regulated by miRNAs [10]. More and more studies showed that miRNAs play important roles in gastric cancer progression [11-13]. Through bioinformatic search, we identified miR-302b was one of the most significantly downregulated miRNAs in GC cells [14], which is consistent with a study conducted by Khalili et al [15]. They also show that miR-302b is downregulated in high-grade gastric cancer. Further, we showed that miR-302b inhibits tumorigenesis by targeting EphA2 through Wnt/ $\beta$-catenin/EMT pathway in gastric cancer cells and mice model [14].

In this study, we'd like to investigate the clinical significance of the mRNA and protein expression level of miR-302b and EphA2 in gastric cancer. We demonstrate that the expression of $\mathrm{miR}-302 \mathrm{~b}$ was negatively correlated with the expression of EphA2, and was the dependent prognostic factor for GC patients. Thus, our results provide a potential epigenetic target for potential gastric cancer therapies that intervene with EphA2.

\section{Materials and Methods}

\section{Antibodies and reagents}

Primary antibodies for EphA2 (\#6997, diluted 1: 1000), and GAPD-H (\#2118, diluted 1:1000) were purchased from Cell Signaling technology MA, USA.

\section{Real-time reverse transcriptase polymerase chain reaction (Real-time $\mathbf{R T}$-PCR)}

Total RNA was extracted using Trizol (Invitrogen, Carlsbad, CA, USA) according to the manufacturer's instructions. The cDNA was synthesized using $1 \mu \mathrm{g}$ of total RNA and TaqMan Reverse Transcription Reagents (Applied Biosystems, Foster City, CA, USA). The primers for EphA2 were: forward: 5'-GAGAAGGATGGCGAGTTCAG-3'; reverse: 5'- TCAGACACCTTGCAGACCAG-3'. The relative levels of target genes' mRNA were expressed as the ratio of target to $\beta$-actin and calculated from the standard curve as directed. All reported results are the average ratios of three different independent experiments.

\section{Western blot}

Whole cell extracts were prepared using $0.14 \mathrm{M}$ $\mathrm{NaCl}, \quad 0.2 \mathrm{M}$ triethanolamine, $0.2 \%$ sodium deoxycholate, $0.5 \%$ Nonidet P-40 and supplemented with a protease inhibitor (Sigma-Aldrich). Fifty micrograms of protein lysate was loaded into each well lysates were resolved by SDS-PAGE on $10 \%$ polyacrylamide gels, and then they were transferred to nitrocellulose or PVDF membranes. After blocking with $5 \%$ milk, the transferred membranes were subsequently incubated overnight at $4^{\circ} \mathrm{C}$ with primary antibody, followed by secondary antibody for $1 \mathrm{~h}$ at routine temperature. Bands were visualized using an ECL Advance Detection System (Amersham Biosciences, Piscataway, NJ, USA).

\section{Human samples and preparation}

In total, 125 GC tissues and the corresponding adjacent, non-tumorous gastric tissues were obtained from the Department of Gastrointestinal Surgery, Xiangya Hospital, Central South University (Changsha,China), between January 2015 and December 2016. mRNAs were extracted from all tissues. Before surgical resection, GC was confirmed in all patients by a certified pathologist. No patient had received neoadjuvant chemotherapy or radiotherapy before radical gastrectomy. Resected tissues were either 1) immediately frozen in liquid nitrogen and stored at $-80^{\circ} \mathrm{C}$ for RNA extraction, or 2) fixed in $37 \%$ formaldehyde solution for paraffin embedding.

Then more patients were included for further analysis. From January 2011 to December 2012, 898 patients with newly were diagnosed gastric cancer at Xiangya hospital. The inclusion criteria were as follows: (1) histologically confirmed diagnosis of gastric adenocarcinoma; (2) availability of clinical data of surgery, chemotherapy and survival; (3) All patients with stage IV who were treated with more than two lines platinum-based chemotherapy. Of the 898 patients screened, 226 patients fulfilled the inclusion criteria and were recruited in the training cohort. The validation cohort of 128 patients with stage IV was recruited at Hunan Cancer Hospital and The Affiliated Cancer Hospital of Xiangya School of Medicine, Central South University (Changsha, China) from February 2013 to January 2015. The inclusion criteria were the same as those previously described. The cancer tissues were excised and fixed in 10\% neutral-buffered formalin, and then they were embedded in paraffin blocks.

All samples were collected and used after obtaining informed consent. This study was approved by the Research Ethics Committees of Xiangya Hospital, Central South University, and Hunan 
Cancer Hospital and The Affiliated Cancer Hospital of Xiangya School of Medicine, Central South University.

\section{In situ hybridization}

A double-DIG-labeled, locked, nucleic acid, microRNA probe complementary to hsa-miR-302b (610016-360; Exiqon, Vedbaek, Denmark) was designed for ISH conforming to the manufacturer's instructions. A scrambled miRNA probe was used as negative control. In addition, 4 - $\mu$ m-thick tissue sections were deparaffinized in xylene and ethanol, incubated with Proteinase-K $(15 \mu \mathrm{g} / \mathrm{ml}, 10 \mathrm{~min}$ at $\left.37^{\circ} \mathrm{C}\right)$, washed twice in PBS, dehydrated in ethanol, hybridized $\left(1 \mathrm{~h}\right.$ at $\left.49^{\circ} \mathrm{C}\right)$ with hybridization mix (1:500 dilution of probe), washed in standard saline citrate buffer, and blocked. Subsequently, staining was developed with sheep anti-digoxin-horseradish peroxidase antibody and visualized with diaminobenzidine, followed by hematoxylin counter staining of the nuclei. The results were evaluated independently by two experienced pathologists. In brief, 10 random visual fields for each section of a sample were selected randomly. The expression intensity scores (0 points, $0-5 \%$; 1 point, $6-25 \%$; 2 points, $26-50 \%$; 3 points more than $50 \%$ ) and positive staining cell scores ( 1 point, weak intensity; 2 points, moderate intensity; 3 points, strong intensity) were added. Total scores $\geq 3$ points indicated tissues with significant overexpression and noted as "positive" to simplify data analysis.

\section{Immunohistochemistry}

The paraffin-embedded tumors were cut into $0.25-\mu \mathrm{m}$-thick sections, and then they were de-paraffinizedand rehydrated. After proteolytic digestion, the slides were blocked with 2.5\% hydrogen peroxide in methanol for $30 \mathrm{~min}$ at room temperature, and then incubated with primary antibody overnight at $4^{\circ} \mathrm{C}$. After washing, the slices were incubated with peroxidase-labeled polymer and substrate chromogen. Finally, sections were incubated in phosphate-buffered solution containing diaminobenzidine for $5 \mathrm{~min}$ at room temperature. Negative controls were prepared by substituting primary antibody with non-immune rabbit serum. Two independent pathologists evaluated and scored the sections in 10 randomly selected visual fields for each section (double-blinded). The evaluation of staining result was graded semi-quantitatively as described previously in in situ hybridization. The expression intensity scores (0 points, $0-5 \%$; 1 point, $6-25 \%$; 2 points, $26-50 \%$; 3 points, more than $50 \%$ ) and positive staining cell scores (1 point, weak intensity; 2 points, moderate intensity; 3 points, strong intensity) were added. Total scores $\geq 3$ points indicated tissues with significant overexpression and were noted as "high" to simplify data analysis, and "low" refers to scores $<3$ points.

\section{Statistical analysis}

Results are expressed as mean \pm SEM from at least three independent experiments. Using the GraphPad Prism statistical program, data were analyzed using Student's t-test, unless otherwise specified (Mann-Whitney test, ROC, Pearson correlation, etc.). Two-tailed $\mathrm{P}$ values less than 0.05 were considered significant. Statistical analyses were performed using IBM SPSS22.0 software (SPSS). The EphaA2 and miR-302b expression levels and clinicopathological parameters were compared by the Pearson $\chi^{2}$-test or Fisher exact test. Bivariate correlations between study variables were calculated by Spearman's rank correlation coefficients. Overall survival time was calculated using the Kaplan-Meier method, and differences between groups were assessed using the log-rank test. Multivariate analyses were calculated by the Cox proportional hazards regression model. $P<0.05$ was considered to be statistically significant.

\section{Results}

\section{mRNA expression level of miR-302b is correlated inversely with EphA2 in GC patients}

To investigate whether miR-302b and EphA2 have clinical significance, we first measured the mRNA level of miR-302b and EphA2 by real-time PCR analysis in cancer tissues and adjacent normal gastric mucosa of 125 patients with GC after radical resection surgery at Xiangya Hospital, Centre South University. miR-302b expression levels were clearly downregulated in GC tissues (Figure 1A), while EphA2 expression levels were upregulated in these tissues (Figure 1B). Pearson's correlation analysis revealed a significant inverse correlation between miR-302b and EphA2 expression levels, as shown in Figure $1 C(r=-0.4209, P<0.0001)$.

To determine the prognostic significance of miR-302b and/or EphA2 expression in GC patients, we choose 226 GC patients with complete clinicopathological and long-term follow-up data available at Xiangya hospital. ISH was performed to test the abundance of miR-302b, and immunohistochemistry (IHC) was performed to analyze protein levels of EphA2. The patients' clinicopathological features are listed in Table 1. Representative samples showing expression levels of miR-302b by ISH and EphA2 by IHC in patients with GC and the surrounding, non-cancerous tissues (Figure 2). 


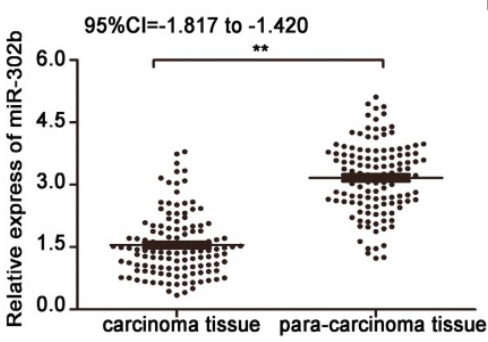

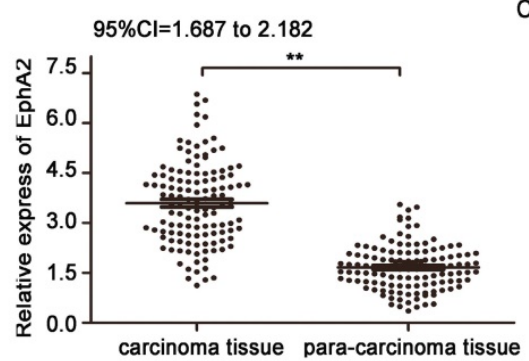

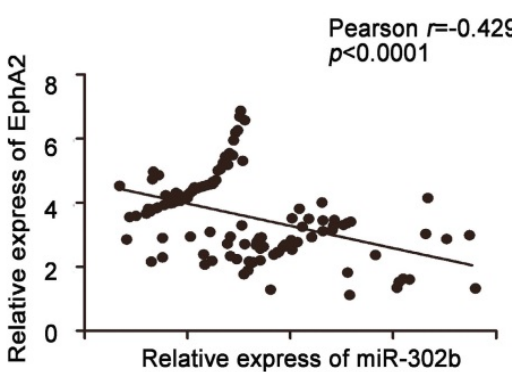

Figure 1. The expression and inverse correlation of miR-302b and EphA2 in gastric cancer tissues.A and B.qRT-PCR was used to detect the expression of miR-302b(A) and EphA2(B) in 125 gastric carcinoma tissues and the adjacent non-cancerous tissues. (C) Pearson's correlation analysis was employed to evaluate the correlation between the expression of miR-302b and EphA2. $* * P<0.01$.

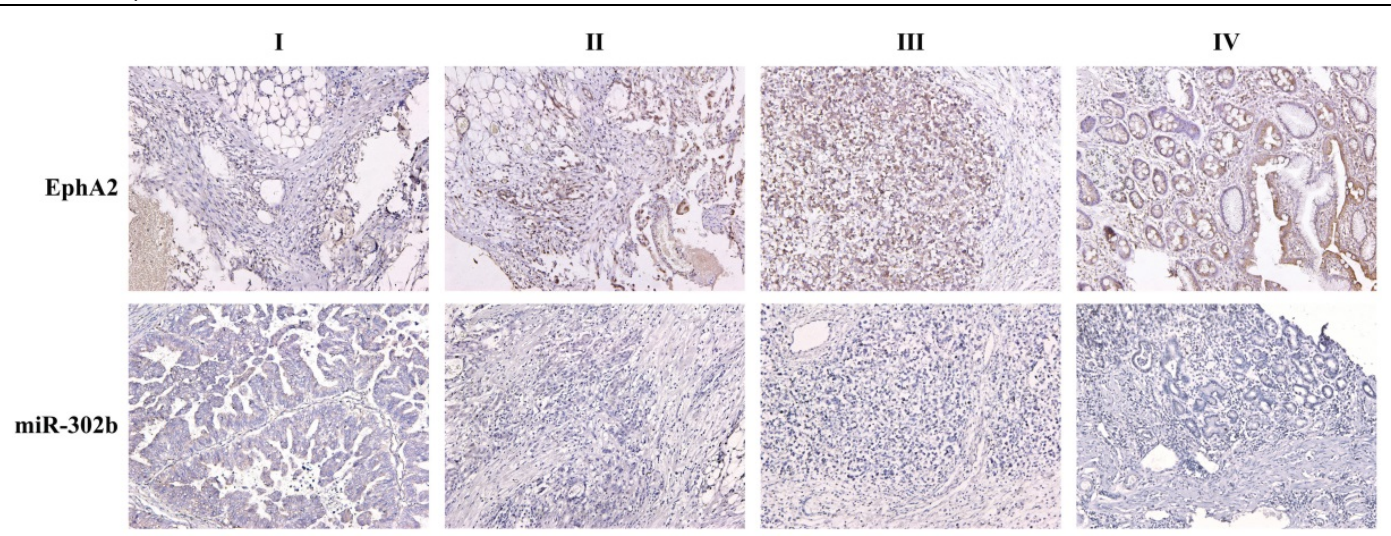

Figure 2. Representative samples showing expression levels of miR-302b by in situ hybridization (ISH) and EphA2 by immunohistochemistry (IHC) in patients with stage I through IV GC and the surrounding, non-cancerous tissue. Scale bars $=100 \mu \mathrm{m}$.

Table 1. The correction between the expression of miR-302b, EphA2 and the clinicopathologic characteristics of patients in the training cohort.

\begin{tabular}{|c|c|c|c|c|c|c|c|}
\hline \multirow[t]{2}{*}{ Variable } & \multirow[t]{2}{*}{$\mathrm{n}$} & \multicolumn{3}{|c|}{ EphA2 } & \multicolumn{3}{|c|}{ miR302b } \\
\hline & & High & Low & $P$ & High & Low & $P$ \\
\hline \multicolumn{8}{|l|}{ Age (years) } \\
\hline$<60$ & 123 & 89 & 34 & 0.684 & 54 & 69 & 0.128 \\
\hline$\geqq 60$ & 103 & 77 & 26 & & 35 & 68 & \\
\hline \multicolumn{8}{|l|}{ Gender } \\
\hline Male & 113 & 86 & 27 & 0.366 & 45 & 68 & 0.892 \\
\hline Female & 113 & 80 & 33 & & 44 & 69 & \\
\hline \multicolumn{8}{|l|}{$\begin{array}{l}\text { Primary tumor } \\
\text { location }\end{array}$} \\
\hline Fundus and cardia & 110 & 85 & 25 & 0.205 & 37 & 73 & 0.085 \\
\hline Distal third & 116 & 81 & 35 & & 52 & 64 & \\
\hline \multicolumn{8}{|l|}{ Lauren's } \\
\hline Diffuse & 131 & 94 & 37 & 0.498 & 47 & 84 & 0.206 \\
\hline Intestinal & 95 & 72 & 23 & & 42 & 53 & \\
\hline \multicolumn{8}{|l|}{ Depth of invasion } \\
\hline $\mathrm{T} 1$ & 22 & 12 & 10 & 0.036 & 15 & 7 & 0.003 \\
\hline $\mathrm{T}_{2}$ & 55 & 37 & 18 & & 27 & 28 & \\
\hline $\mathrm{T}_{3}$ & 118 & 90 & 28 & & 39 & 79 & \\
\hline $\mathrm{T}_{4}$ & 31 & 27 & 4 & & 8 & 23 & \\
\hline \multicolumn{8}{|l|}{$\begin{array}{l}\text { Lymph node } \\
\text { metastasis }\end{array}$} \\
\hline Positive & 136 & 110 & 26 & 0.002 & 43 & 93 & 0.003 \\
\hline Negative & 90 & 56 & 34 & & 46 & 44 & \\
\hline \multicolumn{8}{|l|}{ TNM stage } \\
\hline I & 23 & 10 & 13 & $<0.001$ & 16 & 7 & $<0.001$ \\
\hline II & 52 & 33 & 19 & & 31 & 21 & \\
\hline III & 67 & 52 & 15 & & 19 & 48 & \\
\hline IV & 84 & 71 & 13 & & 23 & 61 & \\
\hline \multicolumn{8}{|l|}{ Lymphatic invasion } \\
\hline Yes & 97 & 77 & 20 & 0.002 & 32 & 65 & 0.001 \\
\hline No & 81 & 47 & 34 & & 46 & 35 & \\
\hline \multicolumn{8}{|l|}{ Venous invasion } \\
\hline Yes & 56 & 43 & 13 & 0.161 & 22 & 34 & 0.409 \\
\hline No & 122 & 81 & 41 & & 56 & 66 & \\
\hline
\end{tabular}

Table 2. The correlation between protein expression levels of EphA2 and miR-302b in the training and validation cohorts of gastric cancer tissues.

\begin{tabular}{lllll}
\hline Sets & \multicolumn{2}{l}{ EphA2 } & & \\
\cline { 2 - 4 } & High & Low & $r$ & $P$ \\
\hline $\begin{array}{l}\text { Training set (Stage I-IV) } \\
\text { miR302b }\end{array}$ & & & -0.336 & $<0.001$ \\
$\begin{array}{l}\text { High } \\
\text { Low }\end{array}$ & 49 & 40 & & \\
Training set (Stage IV) & & 20 & & \\
miR302b & & & & \\
High & 15 & 8 & -0.328 & 0.005 \\
Low & 56 & 5 & & \\
Validation set(Stage IV) & & & & \\
miR302b & & & -0.312 & $<0.001$ \\
High & 31 & 19 & & \\
Low & 69 & 9 & & \\
\hline
\end{tabular}

In line with the mRNA expression levels of miR-302b and EphA2, the protein expression of them exhibited an inverse correlation in the cancer tissues of GC patients (Table 2, $\mathrm{r}=-0.336, \mathrm{P}<0.001$ ). As shown in Figure 1C (Fig 1C), miR-302b showed markedly low or negative expression in GC tissue from stage I to IV relative to adjacent, normal tissue, while the expression of EphA2 was higher in cancer tissues than in normal tissues. As shown in Table 1, higher EphA2 expression and lower miR-302b expression were each correlated with increased depth of invasion $(\mathrm{P}=0.036$, 0.003 , respectively), lymph node metastasis $(\mathrm{P}=0.002$, 0.003 , respectively), higher clinical stage $(\mathrm{P}<0.001$, $<0.001$, respectively), and lymphatic invasion ( $\mathrm{P}$ 
$=0.002,0.001$, respectively). These data support the hypothesis that inverse expression between miR-302b and EphA2 modulate the invasiveness of tumors in vivo, corroborating with our in vitro data [14].

\section{miR-302b and EphA2 expression level are dependent prognostic factors for GC patients}

Then, we conducted a patient survival analysis with available follow-up information on 84 patients with stage IV GC (a subset of the training cohort of 226 GC patients from Xiangya Hospital, Central South University) to determine the influence of miR-302b and EphA2 on metastatic advanced GC. The Cox univariate regression analysis indicated that peritoneal metastasis, miR-302b and EphA2 expression were prognostic indicators of overall survival (OS) (Table 3: $\mathrm{P}=0.001, \mathrm{P}=0.002, \mathrm{P}<0.001$, respectively), whereas age, gender, liver metastasis, and Lauren's type had no prognostic significance for OS (Table 3, $\mathrm{P}=0.115, P=0.721, P=0.887, P=0.991$, respectively). And these results were in accordance with Cox multivariate regression analysis, which showed that miR-302b expression $(\mathrm{HR}=1.86 ; 95 \% \mathrm{CI}$, 1.11-3.14; $P=0.021)$, EphA2 expression $(\mathrm{HR}=2.84$; $95 \% \mathrm{CI}, 1.34-6.01 ; P=0.006)$ and peritoneal metastasis $(\mathrm{HR}=1.97 ; \quad 95 \% \mathrm{CI}, 1.21-3.22 ; \quad \mathrm{P}=0.006)$ were independent prognostic factors for OS, respectively (Table 4). Then we conducted a Kaplan-Meier analysis on patients based on the expression levels of

A

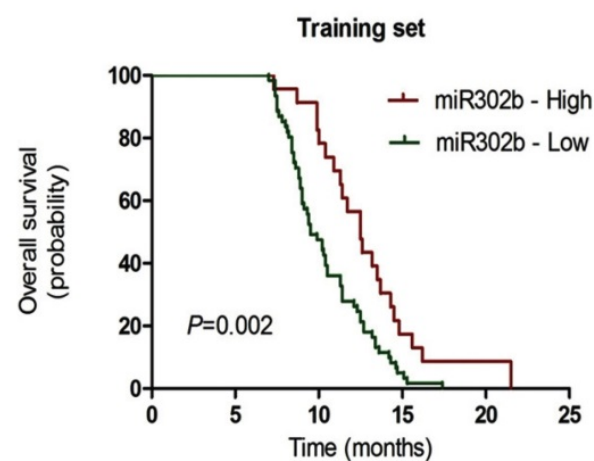

C

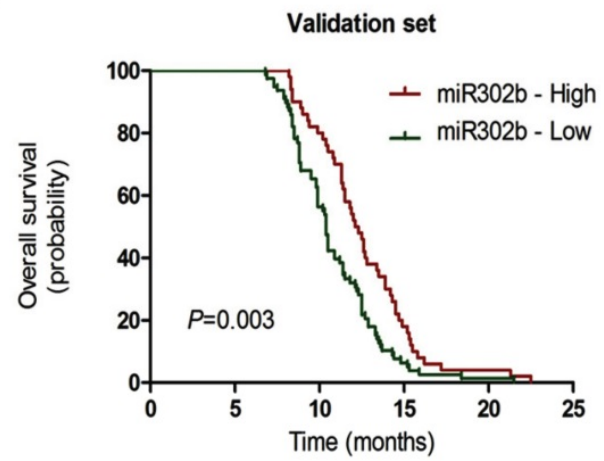

miR-302b or EphA2. We observed that patients with low miR-302b expression had shorter OS than patients with high expression (Figure 3A, P $=0.002$ ). Conversely, high levels of EphA2 expression correlated with shorter OS (Figure 3B, P <0.001).

Table 3. Univariate analyses of the expression of miR-302b, EphA2 with the clinicopathologic characteristics in the training and validation cohorts.

\begin{tabular}{|c|c|c|c|c|c|c|}
\hline \multirow[t]{2}{*}{ Variable } & \multirow[t]{2}{*}{$\mathbf{n}$} & \multicolumn{2}{|l|}{ Training set } & \multirow[t]{2}{*}{ n } & \multicolumn{2}{|c|}{ Validation set } \\
\hline & & $\begin{array}{l}\text { Median OS } \\
\text { (months) }\end{array}$ & $P$ & & $\begin{array}{l}\text { Median OS } \\
\text { (months) }\end{array}$ & $P$ \\
\hline \multicolumn{7}{|l|}{ Age (years) } \\
\hline$<60$ & 42 & 11.4 & 0.115 & 55 & 11.3 & 0.131 \\
\hline$\geq 60$ & 42 & 10.0 & & 73 & 11.3 & \\
\hline \multicolumn{7}{|l|}{ Gender } \\
\hline Male & 42 & 10.4 & 0.721 & 63 & 10.9 & 0.296 \\
\hline Female & 42 & 10.4 & & 65 & 11.5 & \\
\hline \multicolumn{7}{|l|}{ Lauren's } \\
\hline Diffuse & 46 & 10.4 & 0.991 & 76 & 10.5 & 0.055 \\
\hline Intestinal & 38 & 10.4 & & 52 & 12.5 & \\
\hline \multicolumn{7}{|l|}{$\begin{array}{l}\text { Peritoneal } \\
\text { metastasis }\end{array}$} \\
\hline Yes & 29 & 9.5 & 0.001 & 27 & 9.0 & 0.017 \\
\hline No & 55 & 11.4 & & 101 & 11.4 & \\
\hline \multicolumn{7}{|l|}{$\begin{array}{l}\text { Liver } \\
\text { metastasis }\end{array}$} \\
\hline Yes & 23 & 10.2 & 0.887 & 23 & 8.9 & 0.029 \\
\hline No & 61 & 10.5 & & 105 & 11.4 & \\
\hline \multicolumn{7}{|l|}{ EphA2 } \\
\hline High & 71 & 10.0 & $<0.001$ & 100 & 10.4 & 0.009 \\
\hline Low & 13 & 13.2 & & 28 & 12.5 & \\
\hline $\operatorname{miR} 302 b$ & & & 0.002 & & & 0.003 \\
\hline High & 23 & 9.5 & & 78 & 12.1 & \\
\hline Low & 61 & 12.5 & & 50 & 10.4 & \\
\hline
\end{tabular}

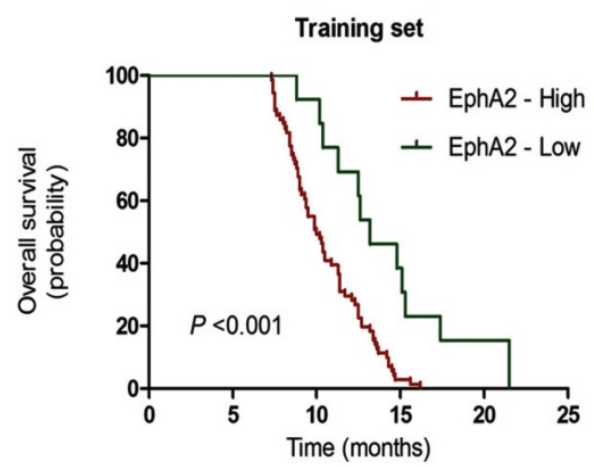

D

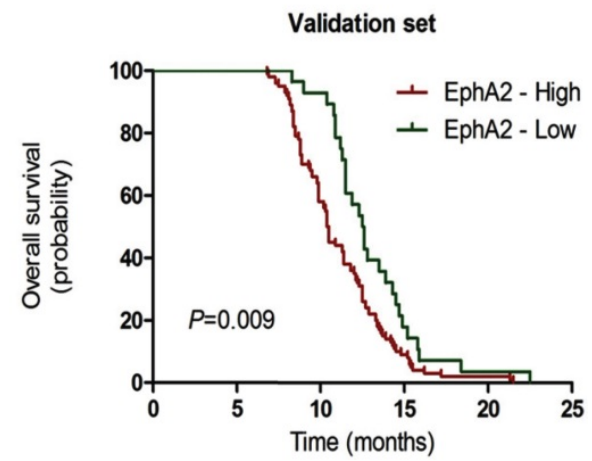

Figure 3. The prognostic significance of miR-302b and EphA2 in stage IV GC patients. The Kaplan-Meier analysis of overall survival for patients with low and high expression of miR-302b and EphA2 groups in the training $(A, B)$ and validation cohorts $(C, D)$. 
Table 4. Multiivariate Cox regression analyses of the expression of miR-302b, EphA2 with the clinicopathologic characteristics in the training and validation cohorts.

\begin{tabular}{llllll}
\hline Variable & Comparison & Training set & \multicolumn{3}{l}{ Validation set } \\
\cline { 3 - 6 } & & HR (95\%CI) & $\boldsymbol{P}$ & HR (95\%CI) & $\boldsymbol{P}$ \\
\hline Liver metastasis & Yes vs. No & $1.11(0.67-1.86)$ & 0.679 & $1.38(0.86-2.21)$ & 0.185 \\
Peritoneal & Yes vs. No & $1.97(1.21-3.22)$ & $\mathbf{0 . 0 0 6}$ & $1.65(1.07-2.54)$ & $\mathbf{0 . 0 2 4}$ \\
metastasis & & & & & \\
EphA2 & High vs. Low & $2.84(1.34-6.01)$ & $\mathbf{0 . 0 0 6}$ & $1.64(1.06-2.54)$ & $\mathbf{0 . 0 2 8}$ \\
miR302b & Low vs. High & $1.86(1.11-3.14)$ & $\mathbf{0 . 0 2 1}$ & $1.51(1.04-2.20)$ & $\mathbf{0 . 0 3 0}$ \\
\hline
\end{tabular}

\section{Validation of the clinical significance of miR-302b and EphA2 expression level for stage IV GC patients}

To further validate the significance of $\mathrm{miR}-302 \mathrm{~b}$ and EphA2 expression, we analyzed the expression of both by the same methods in an independent, validation cohort $(n=128$, from Hunan Cancer Hospital and The Affiliated Cancer Hospital of Xiangya School of Medicine, Central South University) with stage IV gastric cancer patients. Compared with training cohort, Univariate analysis showed an additional factor, liver metastasis, had prognostic significance on OS (Table 3), $\mathrm{P}=0.029$ ), while multivariate analysis displayed no statistical difference between the two groups (Table 4, $\mathrm{P}=0.185$ ). In line with our previous results in the training cohort, miR-302b, EphA2 and peritoneal metastasis were independent prognostic factors for OS $(\mathrm{P}=0.028, \mathrm{P}$ $=0.030, \mathrm{P}=0.024$, respectively, Table 4) in Cox multivariate regression analysis. More importantly, patients with low miR-302b and high EphA2 levels had poor prognosis (Figure $3 \mathrm{C}$ and $3 \mathrm{D}$ ). These results suggest that miR-302b and EphA2 levels are promising prognostic indicators for GC patients in clinical practice.

\section{Discussion}

In this study, we investigate the clinical significance of the expression level of miR-302b and EphA2 in gastric cancer. We demonstrate that the expression of miR-302b was negatively correlated with the expression of EphA2, and miR-302b and EphA2 expression level are dependent prognostic factors for GC patients, which were also validated in a validation cohort.

Expression of EphA2 protein has been reported to increase in various tumors, including breast, prostate, lung, colon, glioblastoma multiforme, ovarian, esophageal, as well as gastric cancer, and this overexpression is correlated with poor survival, which suggests that it's very promising to target EphA2 for further treatment development $[5,7$, 16-21]. In this study, the expression of EphA2 also was higher in gastric cancer tissues than in para-carcinoma tissues, which validated previous researches. The mechanism of the antitumor effects of EphA2 exhibits in cell proliferation, cell cycle, migration, invasion, and EMT of gastric cancer and other cancer types [5, 22-25]. However, the regulation of EphA2 and the cause of its overexpression in GC are still largely unknown.

Several studies have reported that miRNAs can regulate EphA2 in cancer cells [26-28]. Overexpressing miR-200c is reported to inhibit invasion, migration, and vascular tube formation of malignant glioma, breast cancer, and lung carcinoma cells. And these phenotypic changes are associated with EphA2 downregulation [26], furthermore, miR-200a is demonstrated to direct repress EphA2 oncogene in triple-negative breast cancer cells[27]. MiR-26b can also inhibit cell proliferation, migration, and invasion by targeting EphA2 in hepatocellular carcinoma cells [28]. All the miRNAs mentioned above can influence the tumor behavior through regulating EphA2 in different tumors.

The miR-302 cluster consist of six members: miR-302a, miR-302b, miR-302c, miR-302d, and miR-367, and they are the abundantly expressed in human embryonic stem cells (hESCs) and functions in the self-renewal and pluripotency processes[29, 30], which made them also played important roles in tumorigenesis and cancer progression [15, 31, 32].

miR-302b can inhibite cell proliferation and induced apoptosis in malignant pleural mesothelioma cells through targeting Mcl-1[33]. Our previous study has showed that miR-302b was one of the most significant downregulating miRNA of targeting EphA2 in GC cells [14]. Furthermore, we demonstrated that miR-302b serves as a critical suppressor of GC cell tumorigenesis and metastasis by targeting the EphA2/Wnt/ $\beta$-catenin/EMT pathway [14]. So we'd like to investigate the clinical significance of the expression level of miR-302b and EphA2 in gastric cancer.

First, real-time PCR results from 125 patients who underwent radical resection surgery demonstrated that mRNA expression of miR-302b in cancer tissue was significantly lower than that of adjacent normal gastric mucosa. The expression characteristics of miR-302b were in line with previous studies, miR-302b was reported to be downregulated in gastric cancer [15, 31, 32]. However, there were only about 30 patients in those 3 researches, our study apparently has a larger sample number, and the results will be more convincing. As we mentioned above, many studies showed that EphA2 is overexpressed in many tumors, and we confirmed that EphA2 increased in gastric cancer [5, 7, 18-20]. Next, based on the fact that miR-302b suppressed GC cell tumorigenesis and metastasis by targeting EphA2 
[14], we analyzed whether miR302b/EphA2 expression have any clinical significance by performing a correlation analysis between expression levels of these genes and clinicopathological features in the tumor tissues of 226 GC patients. These results indicated that miR-302b levels had an inverse relationship with those of EphA2 in GC samples. The inverse expression between miR-302b and EphA2 were correlated with depth of invasion, lymph node metastasis, clinical stage, and lymphatic invasion, all of which indicated tumor invasiveness and progression. Importantly, low expression of miR-302b and high expression of EphA2 were predictors of poor OS in stage IV GC patients in both tested cohorts, suggesting that miR-302 and EphA2 are promising predictors for GC prognosis clinically. Importantly, our study has training cohort and independent validation cohort, combination of which made our results more solid and conclusion more reliable.

\section{Conclusion}

We demonstrated that miR-302b and EphA2 expression were negatively correlated, and they had prognostic significance on GC in clinic. These data provide novel insights into GC progression and offer miR-302b and EphA2 as potential prognostic factors and therapeutic targets for this disease.

\section{Abbreviations}

GC: Gastric cancer; RTKs: Receptor tyrosine kinases; miRNA: MicroRNAs; H\&E: Hematoxylin and eosin; OS: Overall survival; HR: Hazard ratio; ESC: embryonic stem cell; EMT: epithelialmesenchymal transition; 3'-UTR: 3'-untranslated region; IHC: immunohistochemistry; ISH: In situ hybridization; hESCs: human embryonic stem cells.

\section{Acknowledgements}

\section{Ethics approval and consent to participate}

This study was approved by the Research Ethics Committees of Xiangya Hospital, Central South University, and Hunan Cancer Hospital and The Affiliated Cancer Hospital of Xiangya School of Medicine, Central South University. All samples were collected and used after obtaining informed consent. All animal experiments were performed in strict accordance with the principles and procedures approved by the Committee on the Ethics of Animal Experiments of Central South University.

\section{Funding}

This work was supported by grants from the National Natural Science Foundation of China (81402007, and 81673517), Wujieping medical foundation (320.6750.13132) and Natural Science Foundation of Hunan Province (2018JJ3852).

\section{Competing Interests}

The authors have declared that no competing interest exists.

\section{References}

1. Siegel RL, Miller KD, Jemal A: Cancer statistics, 2016. CA: a cancer journal for clinicians. 2016; 66:7-30.

2. Bang YJ, Van Cutsem E, Feyereislova A, Chung HC, Shen L, Sawaki A Lordick F, Ohtsu A, Omuro Y, Satoh T et al: Trastuzumab in combination with chemotherapy versus chemotherapy alone for treatment of HER2-positive advanced gastric or gastro-oesophageal junction cancer (ToGA): a phase 3, open-label, randomised controlled trial. Lancet. 2010; 376:687-97.

3. Pasquale EB: Eph-ephrin bidirectional signaling in physiology and disease. Cell. 2008; 133:38-52.

4. Janes PW, Nievergall E, Lackmann M: Concepts and consequences of Eph receptor clustering. Seminars in cell \& developmental biology. 2012; 23:43-50.

5. Huang J, Xiao D, Li G, Ma J, Chen P, Yuan W, Hou F, Ge J, Zhong M, Tang Y et al: EphA2 promotes epithelial-mesenchymal transition through the Wnt/beta-catenin pathway in gastric cancer cells. Oncogene. 2014; 33:2737-47.

6. Dunne PD, Dasgupta S, Blayney JK, McArt DG, Redmond KL, Weir JA, Bradley CA, Sasazuki T, Shirasawa S, Wang T et al: EphA2 Expression Is a Key Driver of Migration and Invasion and a Poor Prognostic Marker in Colorectal Cancer. Clin Cancer Res. 2016; 22:230-42.

7. Hou F, Yuan W, Huang J, Qian L, Chen Z, Ge J, Wu S, Chen J, Wang J, Chen Z: Overexpression of EphA2 correlates with epithelial-mesenchymal transition-related proteins in gastric cancer and their prognostic importance for postoperative patients. Med Oncol. 2012; 29:2691-700.

8. Yuan WJ, Ge J, Chen ZK, Wu SB, Shen H, Yang P, Hu B, Zhang GW, Chen ZH: Over-expression of EphA2 and EphrinA-1 in human gastric adenocarcinoma and its prognostic value for postoperative patients. Dig Dis Sci. 2009; 54:2410-7.

9. Yuan W, Chen Z, Chen Z, Wu S, Guo J, Ge J, Yang P, Huang J: Silencing of EphA2 inhibits invasion of human gastric cancer SGC-7901 cells in vitro and in vivo. Neoplasma. 2012; 59:105-13.

10. Filipowicz $\mathrm{W}$, Bhattacharyya $\mathrm{SN}$, Sonenberg $\mathrm{N}$ : Mechanisms of post-transcriptional regulation by microRNAs: are the answers in sight? Nature reviews Genetics. 2008; 9:102-14.

11. Hao NB, He YF, Li XQ, Wang K, Wang RL: The role of miRNA and lncRNA in gastric cancer. Oncotarget. 2017.

12. Kurashige J, Mima K, Sawada G, Takahashi Y, Eguchi H, Sugimachi K, Mori M, Yanagihara K, Yashiro M, Hirakawa K et al: Epigenetic modulation and repression of miR-200b by cancer-associated fibroblasts contribute to cancer invasion and peritoneal dissemination in gastric cancer. Carcinogenesis. 2015; 36:133-41.

13. Li R, Yuan W, Mei W, Yang K, Chen Z: MicroRNA 520d-3p inhibits gastric cancer cell proliferation, migration, and invasion by downregulating EphA2 expression. Mol Cell Biochem. 2014; 396:295-305.

14. Huang J, He Y, McLeod HL, Xie Y, Xiao D, Hu H, Chen P, Shen L, Zeng S, Yin $\mathrm{X}$ et al: miR-302b inhibits tumorigenesis by targeting EphA2 via Wnt/ beta-catenin/EMT signaling cascade in gastric cancer. BMC cancer. 2017; $17: 886$.

15. Khalili M, Sadeghizadeh M, Ghorbanian K, Malekzadeh R, Vasei M, Mowla SJ: Down-regulation of miR-302b, an ESC-specific microRNA, in Gastric Adenocarcinoma. Cell J. 2012; 13:251-8.

16. Teoh DG, Secord AA: Antiangiogenic therapies in epithelial ovarian cancer. Cancer control : journal of the Moffitt Cancer Center. 2011; 18:31-43.

17. Herath NI, Boyd AW: The role of Eph receptors and ephrin ligands in colorectal cancer. Int J Cancer. 2010; 126:2003-11.

18. Stammes MA, Prevoo HA, Ter Horst MC, Groot SA, Van de Velde CJ, Chan $\mathrm{AB}$, de Geus-Oei LF, Kuppen PJ, Vahrmeijer AL, Pasquale EB et al: Evaluation of EphA2 and EphB4 as Targets for Image-Guided Colorectal Cancer Surgery. International journal of molecular sciences. 2017; 18.

19. Lee HY, Mohammed KA, Kaye F, Moudgil BM, Nasreen N: EphA2 targeted intratumoral therapy for non-small cell lung cancer using albumin mesospheres. Am J Transl Res. 2017; 9:3293-303.

20. Song W, Hwang $Y$, Youngblood VM, Cook RS, Balko JM, Chen J, Brantley-Sieders DM: Targeting EphA2 impairs cell cycle progression and growth of basal-like/triple-negative breast cancers. Oncogene. 2017; 36:5620-30.

21. Wykosky J, Gibo DM, Stanton C, Debinski W: EphA2 as a novel molecular marker and target in glioblastoma multiforme. Mol Cancer Res. 2005; 3:541-51.

22. Brannan JM, Dong W, Prudkin L, Behrens C, Lotan R, Bekele BN, Wistuba I, Johnson FM: Expression of the receptor tyrosine kinase EphA2 is increased in smokers and predicts poor survival in non-small cell lung cancer. Clin Cancer Res. 2009; 15:4423-30. 
23. Cui XD, Lee MJ, Yu GR, Kim IH, Yu HC, Song EY, Kim DG: EFNA1 ligand and its receptor EphA2: potential biomarkers for hepatocellular carcinoma. Int J Cancer. 2010; 126:940-9.

24. Li X, Wang L, Gu JW, Li B, Liu WP, Wang YG, Zhang X, Zhen HN, Fei Z: Up-regulation of EphA2 and down-regulation of EphrinA1 are associated with the aggressive phenotype and poor prognosis of malignant glioma. Tumour Biol. 2010; 31:477-88

25. Merritt WM, Kamat AA, Hwang JY, Bottsford-Miller J, Lu C, Lin YG, Coffey D, Spannuth WA, Nugent E, Han LY et al: Clinical and biological impact of EphA2 overexpression and angiogenesis in endometrial cancer. Cancer Biol Ther. 2010; 10:1306-14.

26. Koo T, Cho BJ, Kim DH, Park JM, Choi EJ, Kim HH, Lee DJ, Kim IA: MicroRNA-200c increases radiosensitivity of human cancer cells with activated EGFR-associated signaling. Oncotarget. 2017; 8:65457-68.

27. Tsouko E, Wang J, Frigo DE, Aydogdu E, Williams C: miR-200a inhibits migration of triple-negative breast cancer cells through direct repression of the EPHA2 oncogene. Carcinogenesis. 2015; 36:1051-60.

28. Li H, Sun Q, Han B, Yu X, Hu B, Hu S: MiR-26b inhibits hepatocellular carcinoma cell proliferation, migration, and invasion by targeting EphA2. Int $\mathrm{J}$ Clin Exp Pathol. 2015; 8:4782-90.

29. Barroso-del Jesus A, Lucena-Aguilar G, Menendez P: The miR-302-367 cluster as a potential stemness regulator in ESCs. Cell Cycle. 2009; 8:394-8.

30. Barroso-delJesus A, Romero-Lopez C, Lucena-Aguilar G, Melen GJ, Sanchez L, Ligero G, Berzal-Herranz A, Menendez P: Embryonic stem cell-specific miR302-367 cluster: human gene structure and functional characterization of its core promoter. Mol Cell Biol. 2008; 28:6609-19.

31. Liu FY, Wang LP, Wang Q, Han P, Zhuang WP, Li MJ, Yuan H: miR-302b regulates cell cycles by targeting CDK2 via ERK signaling pathway in gastric cancer. Cancer Med. 2016.

32. Khalili M, Vasei M, Khalili D, Alimoghaddam K, Sadeghizadeh M, Mowla SJ: Downregulation of the Genes Involved in Reprogramming (SOX2, c-MYC, miR-302, miR-145, and P21) in Gastric Adenocarcinoma. J Gastrointest Cancer. 2015; 46:251-8.

33. Khodayari N, Mohammed KA, Lee H, Kaye F, Nasreen N: MicroRNA-302b targets Mcl-1 and inhibits cell proliferation and induces apoptosis in malignant pleural mesothelioma cells. Am J Cancer Res. 2016; 6:1996-2009. 